\title{
Adaptation of antiretroviral therapy in human immunodeficiency virus infection with central nervous system involvement
}

\author{
Matthias Mehling, ${ }^{1}$ Henning Drechsler, ${ }^{2,3}$ Jens Kuhle, ${ }^{1}$ Martin Hardmeier, ${ }^{1}$ Ruediger Doerries, ${ }^{4}$ \\ Stephan Ruegg, ${ }^{1}$ and Achim Gass ${ }^{1}$ \\ ${ }^{1}$ Department of Neurology and ${ }^{2}$ Department of Internal Medicine, University Hospital of Basel, Switzerland \\ ${ }^{3}$ Division of Infectious Diseases, University of Texas Southwestern, Dallas, Texas, USA \\ ${ }^{4}$ Department of Virology, University Hospital of Mannheim, Germany
}

\begin{abstract}
The authors describe a patient with known human immunodeficiency virus (HIV)-1 infection who presented with two generalized seizures and was found to have extensive white matter disease and a left/bilateral temporo-occipital focal slowing on electroencephalography (EEG). There were no magnetic resonance imaging (MRI) or cerebrospinal fluid (CSF) indications for opportunistic infection. Plasma viremia was controlled, whereas viral replication was uncontrolled in CSF. CSF-specific genotype-guided adaptation of the antiretroviral therapy in order to optimize central nervous system (CNS) penetration resulted in clinical improvement and normalization of MRI and EEG. Our case report illustrates the importance of individualized antiretroviral therapy in HIV infected patients with neurological complications. Journal of NeuroVirology (2008) 14, 78-84.
\end{abstract}

Keywords: antiretroviral therapy CNS; CNS infection; HAART; HIV; HIV CNS; HIV epilepsy

\section{Case report}

In August 2003, a 40-year-old man with longstanding human immunodeficiency virus (HIV)-1 infection presented with a first-generalized tonic-clonic seizure. HIV-infection was known since 1987 and the patient was enrolled in a methadone program since 1986. He took zidovudine monotherapy from 1993 to1997, and was on highly active antiretroviral therapy (HAART) since 1997. From 1997 to 2003 he was treated with lamivudine and stavudine complemented with indinavir from 1997 to 1999, and nelfinavir from 1999 to 2003 (Figure 1). He also had a brief exposure to efavirenz, which was stopped because of neuropsychiatric side effects. The patient's nadir CD4+ T-cell count was 166/ $\mu \mathrm{l}(11 \%)$ before HAART initiation; but his CD4+ T cells rose

Address correspondence to Matthias Mehling, MD, Department of Neurology, University Hospital Basel, Petersgraben 4, CH-4031 Basel, Switzerland. E-mail: mehlingm@uhbs.ch

Received 23 July 2007; revised 17 September 2007; accepted 10 October 2007. rapidly and remained $>400 / \mu \mathrm{l}$ since 1998 whereas his viremia remained only partially controlled (always $<1000$ copies/ml from 1997 to 2004). He never experienced any opportunistic infection or malignancy. HIV plasma genotyping in 2003 revealed the presence of the D30N and N88D/N mutation, which mediate resistance to nelfinavir and the M184V and T215Y mutation conferring resistance to lamivudine and partial resistance to stavudine. However, because his viral load was always $<1000$ copies, the patient chose to remain on his HAART regimen. At the time of the first seizure, the patient's CD4+ Tcell count was $794 / \mu \mathrm{l}(14 \%)$ and his plasma viral load was 281 copies $/ \mathrm{ml}$. Magnetic resonance imaging (MRI) revealed diffuse frontal and temporal white matter T2-hyperintensities. Contrast-enhanced studies were negative. These findings were consistent with HIV-1-associated encephalopathy and not suggestive for opportunistic infection. The patient remained otherwise asymptomatic and did not show any signs of cognitive impairment. Twelve months later his HAART regimen was changed for more convenient dosing to once-daily ritonavir/atazanavir, 


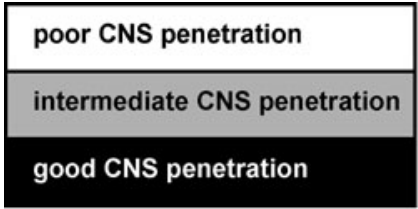

\begin{tabular}{|c|c|c|}
\hline & $2^{\text {nd }}$ seizure & MRI: \\
\hline $1^{\text {st }}$ seizure & $\begin{array}{l}\text { MIRI: worsening white } \\
\text { matter disease; }\end{array}$ & $\begin{array}{l}\text { resolution of white } \\
\text { matter disease; }\end{array}$ \\
\hline MRI: white & CSF HIV-VL: & CSF HIV-VL: \\
\hline matter disease; & 8896 copies $/ \mathrm{mL}$ & $<50$ copies $/ \mathrm{mL}$ \\
\hline Plasma HIV-VL: & Plasma HIV-VL: & Plasma HIV-VL: \\
\hline 281 copies/mL & $<40$ copies $/ \mathrm{mL}$ & $<40$ copies $/ \mathrm{mL}$ \\
\hline
\end{tabular}

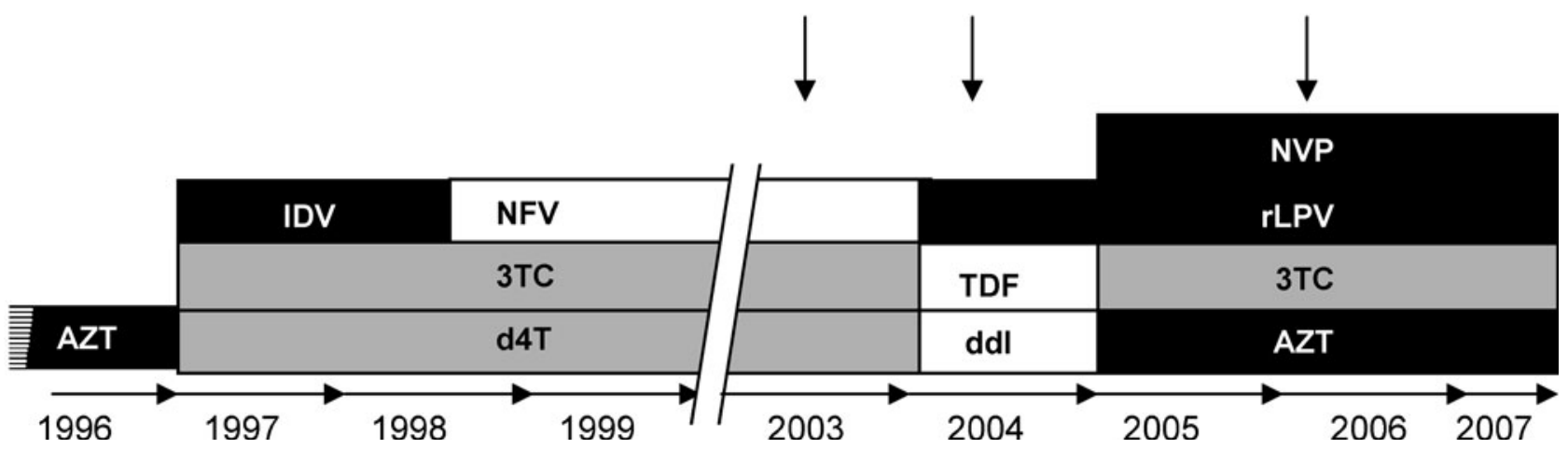

Figure 1 Chronology of antiretroviral therapy with quantification of CNS penetration. VL: viral load. AZT $=$ zidovudine; d4T $=$ stavudine; 3TC = lamivudine; IDV = indinvavir; NFV = nelfinavir; rATV = ritonavir-boosted atazanavir; TDF = tenofovir; ddI = didanosine; LPV = ritonavir-boosted lopinavir; NVP = nevirapine.

with didanosine and tenofovir. His plasma viral load now became undetectable $(<40$ copies $/ \mathrm{ml})$. Two months later he experienced another generalized seizure, which prompted a neurological evaluation, which was unremarkable without signs of cognitive or memory impairment. Repeat MRI showed moderate progression of the diffuse white matter disease (Figure 2A). Electroencephalography (EEG) revealed subtle background alterations with a bilateral temporo-occipital focal slowing (Figure 3A).

Cerebrospinal fluid (CSF) analysis revealed pleiocytosis $\left(32 \times 10^{6} / \mathrm{L}\right)$, elevated protein $(917 \mathrm{mg} / \mathrm{L})$, and immunoglobulin G (IgG) $(527 \mathrm{mg} / \mathrm{L})$ and oligoclonal bands. Serologic assays for borreliae, syphilis, herpes simplex virus, and varicella zoster virus (VZV) were negative as well as polymerase chain reaction (PCR) assays for Toxoplasma gondii, JC virus, Epstein Barr virus, cytomegalovirus, and VZV. The cerebrospinal fluid (CSF) HIV viral load was 8896 copies/ml, whereas plasma viremia remained undetectable $(<40$ copies $/ \mathrm{ml})$. Genotyping of the CSF virus revealed thymidine-analogue mutations M41L and $\mathrm{T} 215 \mathrm{Y}$, conferring intermediate resistance to zidovudine and low-level resistance to didanosine and tenofovir, the A98G polymorphism on the reverse transcriptase gene, and L63P polymorphism on the protease gene. Attempting to adapt the patient's HAART for higher efficacy against the HIV quasispecies in central nervous system (CNS) and for optimal CSF penetration, his regimen was now changed to nevirapine, zidovudine and lamivudine, and lopinavir/ritonavir. The patient was also started on antiepileptic therapy with levetiracetam, which he discontinued after 4 months. He has remained seizure free for almost 3 years to date and still shows no sign of cognitive impairment. A followup MRI demonstrated normalization of previous T2hyperintensities with some widening of the lateral ventricles probably indicating resolution of edema, possibly unmasking some degree of subcortical brain atrophy (Figure 2B). A follow-up EEG after 19 months had also almost normalized (Figure 3B). CSF analysis 12 months after switching to CNS-adapted HAART showed resolution of pleiocytosis and reduction in total protein $(679 \mathrm{mg} / \mathrm{L})$ and $\operatorname{IgG}(345 \mathrm{mg} / \mathrm{L})$. The CSF HIV viral load was now undetectable $(<50$ copies $/ \mathrm{ml})$.

\section{Discussion}

Epileptic seizures are a frequent event in HIVinfected patients. Before the ART-era, their annual incidence was estimated up to $11 \%$ and in one study even up to $17 \%$, whereas a reduced frequency with a prevalence of up to $6 \%$ was reported in the ARTera (Dore et al, 1999; Kellinghaus et al, 2007; Levy and Bredesen, 1988; Pascual-Sedano et al, 1999). In almost $50 \%$ of cases, no causal opportunistic disease was identified, suggesting a HIV-related pathogenesis (Langford et al, 2003). The differential diagnosis in HIV-infected patients with seizures comprises infections such as toxoplasmosis, cryptococcal or tuberculous meningitis, and progressive multifocal leukencephalopathy (PML) (Price, 1996). Furthermore, CNS lymphoma and drugs used for treatment of the HIV infection and metabolic alterations such as renal or hepatic failure may predispose 


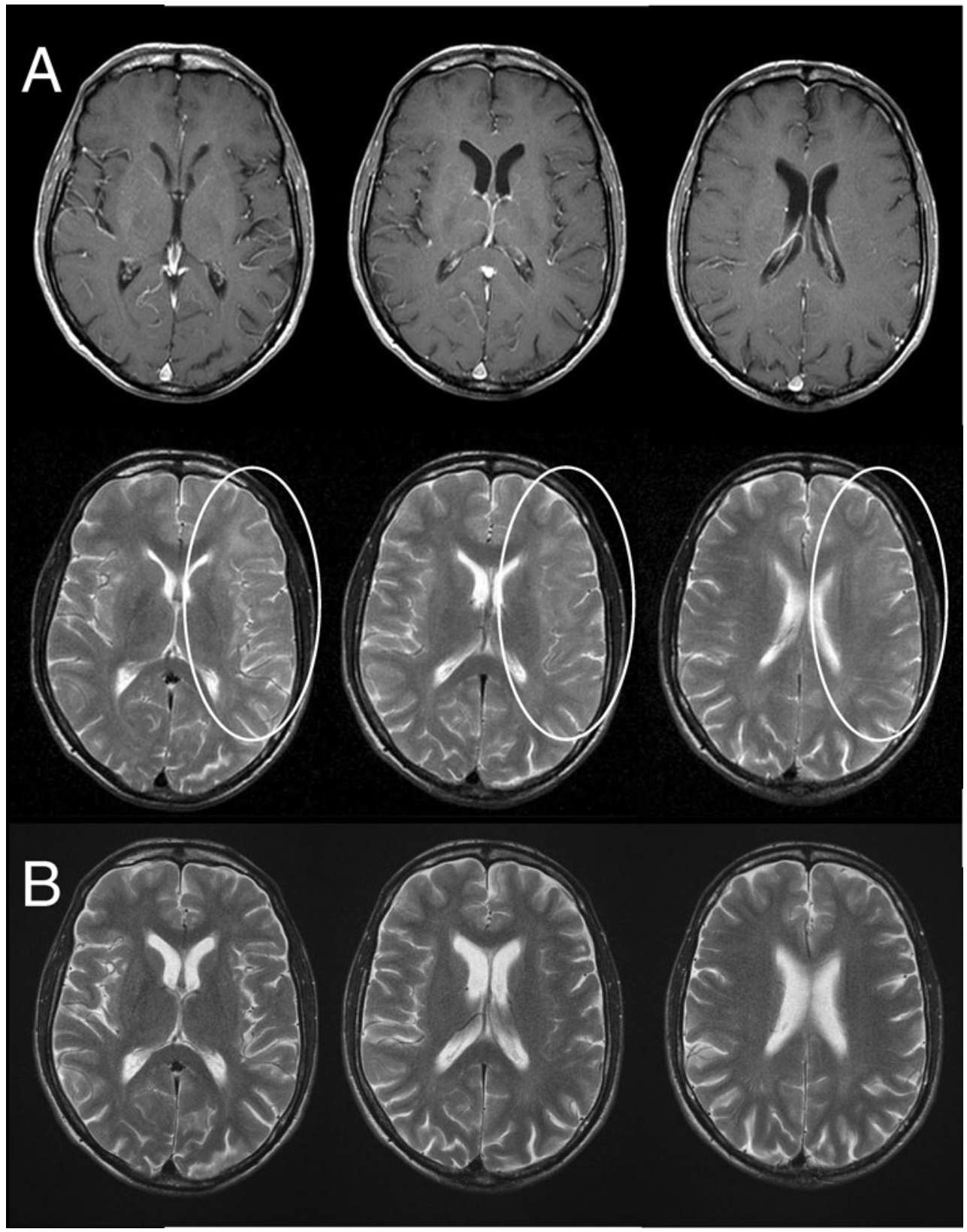

Figure 2 T2-weighted MRI after the second seizure (A) and at 19-month follow-up (B). (A) No contrast-enhancing lesions are detected on T1-weighted images (upper row), whereas mainly subcortical T2-hyperintense white matter abnormality is noted most prominently in left frontal areas (white ellipse; lower row). Normalization of white matter signal intensity and improved differentiation of grey and white matter is demonstrated on follow-up (B).

for seizures (Barton et al, 1992; D’Silva et al, 1995). As the second seizure occurred in our patient after changing the antiretroviral therapy, an immune reconstitution syndrome might have also triggered it. However, no increase of lymphocyte counts was detected after modification of the therapy and the MRI showed no contrast-medium enhancement, both of which argue against the possibility of seizures caused by a immune reconstitution inflammatory syndrome in the CNS (Venkataramana et al, 2006).
We therefore considered the seizures as secondary manifestation of the HIV infection in CNS and initiated an antiepileptic treatment with levetiracetam. Levetiracetam has been reported to be efficacious in HIV-positive patients with HAART treatment (Liedtke et al, 2004). It undergoes minor metabolism by plasma hydrolases, but not by the liver, as it does not interact with the hepatic cytochrome P450 (CYP450) enzyme function by which most protease inhibitors and non-nucleoside reverse transcriptase 

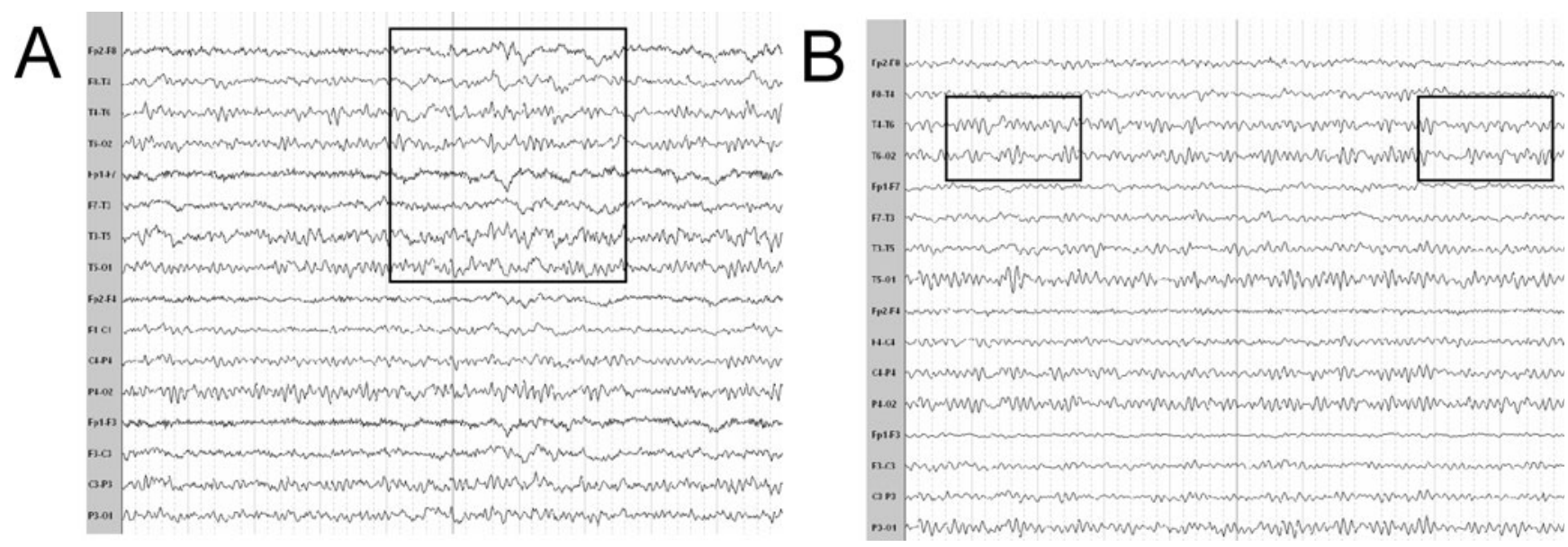

Figure 3 Electroencephalogram after the second seizure (A) and at 19-month follow-up (B). (A) Electroencephalogram (EEG) with modest background alterations and intermittent focal slowing in both temporo-occipital regions, spreading also to the frontal regions (box). (B) Follow-up EEG after adaptation of HAART, which showed almost complete resolution of background alterations and of the left-sided focal slowing whereas a minor slowing still persists on the right temporo-occipital region (boxes).

inhibitors are metabolized. Interactions of antiretroviral agents and the well-known antiepileptic drugs carbamazepine, phenytoin, and phenobarbital have been described in HAART-treated patients, especially when protease inhibitors are given (Liedtke et al, 2004; Bates et al, 2006). Valproic acid has been discussed to have a potential neuroprotective effect in HIV-associated cognitive impairment (Dou et al, 2003; Schifitto et al, 2006). It is metabolized by the hepatic CYP450 system (mainly the 2C9 isoform) and is conjugated by the uridyl-glucuronyl transferase, but has been reported to be save when given in combination with the non-nucleoside reverse transcriptase inhibitor efavirenz and the protease inhibitor lopinavir (DiCenzo et al, 2004). In contrast, valproic acid acts as a modest inhibitor of several CYP450 isoforms and might thereby prolong the elimination half-time of antiretroviral drugs metabolized by this system. Conflicting results regarding a positive effect of valproic acid on the eradication of latent virus reservoirs have been reported (Lehrman et al, 2005; Siliciano et al, 2007). Table 1a comprehensively summarizes the properties of antiepileptic drugs for the treatment of HIV-related seizures with special emphasis given to their potential of interaction with antiretroviral drugs. The hepatic CYP450 metabolism of antiretroviral drugs and their influence on this enzyme system is presented in Table $1 \mathrm{~b}$, which also displays the interaction profile of antiretroviral drug with the currently used antiepileptic drugs.

In HIV-infected patients, imaging studies after seizures have often been reported as negative with respect to structural alterations (McArthur et al, 2005). Clonal expansion of HIV quasispecies in the CNS may account for distinct manifestation of CNS disease and HIV replication in the CNS may be independent from other body compartments as drug mutations distinct from plasma virus can be detected in CSF (Antinori et al, 2005; Temesgen et al, 2006). Furthermore, antiretroviral drug penetration into the CNS varies greatly between different drugs and shows significant interindividual variability (Antinori et al, 2005; McGee et al, 2006). Our patient had been on partially suppressive HAART for 7 years, the last 5 of which he was taking a regimen with overall suboptimal CNS penetration (nelfinavir/stavudine/lamivudine). Even though he had achieved complete suppression of plasma viremia at time of his second seizure after switching his antiretrovirals to a more "modern" regimen, lumbar puncture revealed poorly controlled HIV replication in the CNS. Genotypic resistance testing of the HIV variant isolated from the CSF virus revealed a resistance pattern distinct from plasma virus. We decided to adapt the patient's HAART to specifically target CSF variants, using drugs with optimal CSF/CNS penetration (nevirapine, zidovudine) or proven efficacy in reducing HIV CNS replication (lopinavir/ritonavir), accounting for results of genotypic resistance testing of the patient's HIV CSF variant (Antinori et al, 2005; Thomas, 2004; Van Den Brande, 2006). This was followed by resolution of white matter abnormality on MRI and normalization of EEG. Follow-up CSF studies demonstrated resolved CSF pleiocytosis and undetectable CSF viral load.

This case report highlights the importance of individualizing HAART in patients with HIV associated neurological disease. It also underlines the potential usefulness of measuring CSF viral load with the option of genotyping distinct HIV variants. This diagnostic approach should be considered in all HIVpositive patients who undergo lumbar puncture for CNS disease. It may be of particular interest in patients taking HAART with suboptimal or poor CSF penetration. 
Table 1a Pharmacologic profile of currently used antiepileptic drugs with respect to the use in HIV-infected patients under HAART treatment

\begin{tabular}{|c|c|c|c|c|c|c|}
\hline $\begin{array}{l}\text { Antiepileptic } \\
\text { drug* }^{*}\end{array}$ & $\begin{array}{l}\text { CYP450 } \\
\text { metabolism }\end{array}$ & $\begin{array}{l}\text { Glucuroni- } \\
\text { dation }\end{array}$ & $\begin{array}{l}\text { Protein- } \\
\text { binding }\end{array}$ & $\begin{array}{c}\text { Risk of } \\
\text { interaction }\end{array}$ & $\begin{array}{l}\text { Advantages/ } \\
\text { disadvantages }\end{array}$ & $\begin{array}{l}\text { Use in HIV-patients } \\
\text { under HAART }\end{array}$ \\
\hline Clobazam & $2 \mathrm{C} 19 \gg 3 \mathrm{~A} 4$ & None & $85 \%$ & Modest & $\begin{array}{l}\text { Less sedation than other } \\
\text { BD/dependence }\end{array}$ & Short term: yes \\
\hline Clonazepam & 3A4; inhibits 2B family & None & $86 \%$ & Modest & $\begin{array}{l}\text { Fast + sustained action/ } \\
\text { dependence }\end{array}$ & Emergency use \\
\hline Diazepam & $2 \mathrm{C} 19 \gg 3 \mathrm{~A} 4$ & None & $97-99 \%$ & Modest & $\begin{array}{l}\text { Fast }+ \text { sustained action/ } \\
\text { dependence; several active } \\
\text { metabolites }\end{array}$ & Emergency use \\
\hline Lorazepam & None & Extensive & $93 \%$ & Low & $\begin{array}{l}\text { Fast }+ \text { sustained } \\
\text { action/dependence }\end{array}$ & $\begin{array}{l}\text { Emergency and } \\
\text { short-term use }\end{array}$ \\
\hline Midazolam & $3 \mathrm{~A} 4 / 3 \mathrm{~A} 5$ & $45-57 \%$ & $97 \%$ & Substantial & $\begin{array}{l}\text { Very fast, but short action; } \\
\text { 1:1 antagonist (flumazenil } \\
\text { dependence }\end{array}$ & Emergency use \\
\hline Phenobarbital & $\begin{array}{l}\text { 2C9 } \gg 2 \mathrm{C} 19 \text { and 2E1; } \\
\text { substantial CYP } \\
\text { induction: 2B6, 2B8, 2B9, } \\
\text { 3A4; induction of UGTs }\end{array}$ & $33 \%$ & $55 \%$ & Substantial & $\begin{array}{l}\text { Broad spectrum; efficacious } \\
\text { extremely cheap/cognitive } \\
\text { impairment; altered mood } \\
\text { and behavior; } \\
\text { hepatotoxicity }\end{array}$ & Not recommended \\
\hline Phenytoin & $\begin{array}{l}2 \mathrm{C} 9>2 \mathrm{C} 19 \gg 3 \mathrm{~A} 4 \\
\text { substantial CYP } \\
\text { induction; induction of } \\
\text { UGTs }\end{array}$ & $1 \%$ & $90 \%$ & Substantial & $\begin{array}{l}\text { Broad spectrum; efficacious } \\
\text { cheap/hepatic, bone, and } \\
\text { neurological toxicity; non- } \\
\text { linear pharmacokinetics }\end{array}$ & Not recommended \\
\hline Carbamazepine & $\begin{array}{l}\text { 3A4 > 1A2 and 2C8 } \\
\text { substantial CYP } \\
\text { induction; induction of } \\
\text { UGTs }\end{array}$ & $\sim 50 \%$ & $70-80 \%$ & Substantial & $\begin{array}{l}\text { Very efficacious for focal } \\
\text { seizures; cheap/ } \\
\text { neurological side effects; } \\
\text { liver, bone, blood toxicity }\end{array}$ & Not recommended \\
\hline Valproic acid & $\begin{array}{l}\text { 2A6, 2B6, 2C9, 2C19; } \\
\text { substantial inhibitor of } \\
\text { hepatic CYP enzymes; } \\
\text { inhibition of } \\
\text { glucuronidation }\end{array}$ & $10-70 \%$ & $85-90 \%$ & Modest & $\begin{array}{l}\text { Very broad spectrum; } \\
\text { efficacious; modest } \\
\text { price/hepatic toxicity; } \\
\text { hyperammonemia; } \\
\text { thrombocytopenia; } \\
\text { pancreatitis teratogenicity }\end{array}$ & $\begin{array}{l}\text { Recommended; } \\
\text { additional } \\
\text { ant-retroviral effect }\end{array}$ \\
\hline Lamotrigine & None & $70-80 \%$ & $55 \%$ & Low & $\begin{array}{l}\text { Broad spectrum; efficacious; } \\
\text { strong mood stabilizer/ } \\
\text { long titration phase; skin } \\
\text { rashes; insomnia }\end{array}$ & $\begin{array}{l}\text { Recommended as } \\
\text { second-line drug }\end{array}$ \\
\hline Gabapentin & None & None & None & None & $\begin{array}{l}\text { No interaction; anxiolytic; } \\
\text { effective for neuropathic } \\
\text { pain/restricted } \\
\text { antiepileptic spectrum and } \\
\text { efficacy; elimination } \\
\text { exclusively dependent on } \\
\text { renal function }\end{array}$ & Recommended \\
\hline Topiramate & $\begin{array}{l}\text { Slightly metabolized; weak } \\
\text { inhibition of 2C19; weak } \\
\text { induction of 3A4 }\end{array}$ & $40-70 \%$ & $9-17 \%$ & Low & $\begin{array}{l}\text { Broad spectrum; highly } \\
\text { efficacious/cognitive } \\
\text { effects (memory, } \\
\text { language); weight loss; } \\
\text { slow tritration scheme }\end{array}$ & $\begin{array}{l}\text { Recommended as } \\
\text { second-line drug } \\
\text { (cave: weight loss } \\
\text { and cognitive } \\
\text { decline in HIV } \\
\text { patients) }\end{array}$ \\
\hline Tiagabine & $\begin{array}{l}\text { Extensively by 3A4; neither } \\
\text { induction nor inhibition } \\
\text { of CYP }\end{array}$ & $<10 \%$ & $95 \%$ & Substantial & $\begin{array}{l}\text { Few adverse effects/narrow } \\
\text { antiepileptic spectrum; } \\
\text { modest efficacy; may cause } \\
\text { NCSE }\end{array}$ & $\begin{array}{l}\text { Recommended as } \\
\text { third-line drug }\end{array}$ \\
\hline Oxcarbazepine & $\begin{array}{l}\text { Weak secondary CYP- } \\
\text { dependent metabolism; } \\
\text { mainly CYP-independent } \\
\text { reductases }\end{array}$ & $\sim 70 \%$ & $40 \%$ & Low & $\begin{array}{l}\text { Efficacious; also effective for } \\
\text { neuralgia/hyponatremia }\end{array}$ & Recommended \\
\hline Pregabalin & None & None & None & None & $\begin{array}{l}\text { No interaction; anxiolytic; } \\
\text { effective for neuropathic } \\
\text { pain/restricted } \\
\text { antiepileptic spectrum and } \\
\text { efficacy; elimination } \\
\text { exclusively dependent on } \\
\text { renal function }\end{array}$ & $\begin{array}{l}\text { Recommended; } \\
\text { slightly more } \\
\text { effective than } \\
\text { gabapentin; easier } \\
\text { dosage schedule; } \\
\text { for HIV pat.: weight } \\
\text { gain }\end{array}$ \\
\hline
\end{tabular}


Table 1a Pharmacologic profile of currently used antiepileptic drugs with respect to the use in HIV-infected patients under HAART treatment (Continued)

\begin{tabular}{|c|c|c|c|c|c|c|}
\hline $\begin{array}{l}\text { Antiepileptic } \\
\text { drug* }^{*}\end{array}$ & $\begin{array}{l}\text { CYP450 } \\
\text { metabolism }\end{array}$ & $\begin{array}{l}\text { Glucuroni- } \\
\text { dation }\end{array}$ & $\begin{array}{l}\text { Protein- } \\
\text { binding }\end{array}$ & $\begin{array}{l}\text { Risk of } \\
\text { interaction }\end{array}$ & $\begin{array}{l}\text { Advantages/ } \\
\text { disadvantages }\end{array}$ & $\begin{array}{l}\text { Use in HIV-patients } \\
\text { under HAART }\end{array}$ \\
\hline Levetiracetam & $\begin{array}{l}\text { None (modest metabolism } \\
\text { by plasma hydrolases) }\end{array}$ & None & None & None & $\begin{array}{l}\text { Broad spectrum; efficacious; } \\
\text { fast titration; significant } \\
\text { first-dose effect; almost no } \\
\text { adverse effects/dosage } \\
\text { adaptation required for } \\
\text { impaired kidney function }\end{array}$ & Recommended \\
\hline Zonisamide & $\begin{array}{l}\text { Metabolized by } \\
\text { 3A4 }>2 \text { D6 > 3A5, 2C19; } \\
\text { neither induction nor } \\
\text { inhibitor! }\end{array}$ & $\sim 15 \%$ & $40-60 \%$ & Modest & $\begin{array}{l}\text { Broad spectrum; } \\
\text { efficacious/slow titration }\end{array}$ & $\begin{array}{l}\text { Recommended as } \\
\text { second-line drug }\end{array}$ \\
\hline
\end{tabular}

*Felbamate and vigabatrine not listed because of restricted approval due to their serious side-effect profile.

Abbreviations: CYP: cytochrome P450; HIV: human immunodeficiency virus; NCSE: nonconvulsive status epilepficus; UGT: uridylglucuronid transferase.

Table 1b CYP metabolism of antiretroviral drugs and potential interactions with antiepileptic drugs

\begin{tabular}{|c|c|c|c|c|}
\hline $\begin{array}{l}\text { Antiretroviral } \\
\text { drug }\end{array}$ & $\begin{array}{c}\text { CYP450 enzymes } \\
\text { involved in metbolism }\end{array}$ & $\begin{array}{l}\text { Induction/ } \\
\text { inhibition }\end{array}$ & $\begin{array}{l}\text { Interaction } \\
\text { with } A E D\end{array}$ & $\begin{array}{l}\text { No interaction } \\
\text { to expect with }\end{array}$ \\
\hline \multicolumn{5}{|l|}{ NRTIs } \\
\hline Zidovudine & None (glucuronidation) & None & $\begin{array}{l}\text { CBZ, LZP, PB, PHT, } \\
\text { VPA, (DZP, LTG, } \\
\text { TPM) }\end{array}$ & $\begin{array}{l}\text { CLB, CLN, GBP, LEV, } \\
\text { MDL, OXC, PGB, } \\
\text { TGB, ZOS }\end{array}$ \\
\hline Abacavir & $\begin{array}{l}\text { None (glucuronidation, } \\
\text { alcohol; } \\
\text { dehydrogenase) }\end{array}$ & None & $\begin{array}{l}\text { CBZ, LZP, PB, PHT, } \\
\text { VPA, (DZP, LTG, } \\
\text { TPM) }\end{array}$ & $\begin{array}{l}\text { CLB, CLN, GBP, LEV, } \\
\text { MDL, OXC, PGB, } \\
\text { TGB, ZOS }\end{array}$ \\
\hline Lamivudine & None & None & CBZ, PHT, PB & $\begin{array}{l}\text { CLB, CLN, DZP, GBP, } \\
\text { LEV, LTG, MDL, } \\
\text { OXC, PGB, TGB, } \\
\text { TPM, VPA, ZOS }\end{array}$ \\
\hline Stavudine & None & None & Like lamivudine & Like lamivudine \\
\hline Didanosine & None & None & Like lamivudine & Like lamivudine \\
\hline Zalcitabine & None & None & Like lamivudine & Like lamivudine \\
\hline Tenofovir & None & Very weak $1 \mathrm{~A} 2$ & Like lamivudine & Like lamivudine \\
\hline \multicolumn{5}{|l|}{ NNRTIs } \\
\hline Delavirdine & $3 \mathrm{~A} 4,2 \mathrm{D} 6$ & $\begin{array}{l}\text { 3A4, 2C9, } \\
\quad 2 \mathrm{C} 19 / 3 \mathrm{~A} 4.2 \mathrm{C} 9,2 \mathrm{C} 19\end{array}$ & $\begin{array}{l}\text { CBZ, CLB, CLN, DZP, } \\
\text { MDL, PB, PHT, TGB, } \\
\text { VPA, ZOS, (OXC, } \\
\text { TPM) }\end{array}$ & $\begin{array}{l}\text { GBP, LEV, LTG, LZP, } \\
\text { PGB }\end{array}$ \\
\hline Efavirenz & $3 \mathrm{~A} 4,2 \mathrm{~B} 6$ & $\begin{array}{l}\text { 3A4, 2B6/2B6, } \\
\text { 2C9.2C19, (3A4) }\end{array}$ & $\begin{array}{l}\text { CBZ, CLB, CLN, DZP, } \\
\text { MDL, PB, PHT, TGB, } \\
\text { VPA, ZOS, (OXC, } \\
\text { TPM) }\end{array}$ & $\begin{array}{l}\text { GBP, LEV, LTG, LZP, } \\
\text { PGB }\end{array}$ \\
\hline Nevirapine & $3 \mathrm{~A} 4,2 \mathrm{~B} 6$ & 3A4, 2B6/None & $\begin{array}{l}\text { CBZ, CLN, MDL, PB, } \\
\text { PHT, TGB, ZOS, } \\
\text { (CLB, DZP, OXC, } \\
\text { TPM, VPA) }\end{array}$ & $\begin{array}{l}\text { GBP,LEV, LTG, LZP } \\
\text { PGB }\end{array}$ \\
\hline \multicolumn{5}{|c|}{ स } \\
\hline Ritonavir & $3 \mathrm{~A} 4 \gg 2 \mathrm{D} 6$ & $\begin{array}{l}\text { 1A2, UGTs, } \\
\text { (3A4)/3A4 } \gg 2 \mathrm{D} 6>2 \mathrm{C} 9> \\
\text { 2C19”2A6 > 2E1 } \\
\text { (2B6), 3A5 }\end{array}$ & $\begin{array}{l}\text { CBZ, CLB, CLN, DZP, } \\
\text { LTG, LZP, MDL, OXC, } \\
\text { PB, PHT, TGB, TPM, } \\
\text { VPA, ZOS }\end{array}$ & GBP, LEV, PGB \\
\hline Nelfinavir & $3 \mathrm{~A} 4$ & None/3A4, 2B6, 3A5 & $\begin{array}{l}\text { CBZ, CLN, MDL, PB, } \\
\text { PHT, TGB, VPA, ZOS, } \\
\text { (CLB, DZP, OXC, } \\
\text { TPM) }\end{array}$ & $\begin{array}{l}\text { GBP, LEV, LTG, LZP, } \\
\text { PGB }\end{array}$ \\
\hline Tipranavir & $3 \mathrm{~A} 4$ & $(3 \mathrm{~A} 4) /(3 \mathrm{~A} 4)$ & $\begin{array}{l}\text { CBZ, CLN, MDL, PB, } \\
\text { PHT, TGB, ZOS (CLB, } \\
\text { DZP, OXC, TPM, } \\
\text { VPA) }\end{array}$ & $\begin{array}{l}\text { GBP, LEV, LTG, LZP, } \\
\text { PGB }\end{array}$ \\
\hline Amprenavir & $3 \mathrm{~A} 4$ & None/3A4, 3A5, 2C19 & $\begin{array}{l}\text { CBZ, CLB, CLN, DZP, } \\
\text { MDL, PB, PHT, TGB, } \\
\text { VPA, ZOS, (OXC, } \\
\text { TPM) }\end{array}$ & $\begin{array}{l}\text { GBP, LEV, LTG, LZP, } \\
\text { PGB }\end{array}$ \\
\hline
\end{tabular}


Table 1b CYP metabolism of antiretroviral drugs and potential interactions with antiepileptic drugs (Continued)

\begin{tabular}{|c|c|c|c|c|}
\hline $\begin{array}{l}\text { Antiretroviral } \\
\text { drug }\end{array}$ & $\begin{array}{c}\text { CYP450 enzymes } \\
\text { involved in metbolism }\end{array}$ & $\begin{array}{l}\text { Induction/ } \\
\text { inhibition }\end{array}$ & $\begin{array}{l}\text { Interaction } \\
\text { with } A E D\end{array}$ & $\begin{array}{l}\text { No interaction } \\
\text { to expect with }\end{array}$ \\
\hline Atazanavir & $3 \mathrm{~A} 4$ & None/3A4, UGT1A1 & $\begin{array}{l}\text { CBZ, CLN, LTG, LZP, } \\
\text { MDL, PB, PHT, TGB, } \\
\text { VPA, ZOS, (CLB, } \\
\text { DZP, OXC, TPM) }\end{array}$ & GBP, LEV, PGB \\
\hline Indinavir & $3 \mathrm{~A} 4$ & None/(weak) 3A4 & Like tipranavir & Like tipranavir \\
\hline Saquinavir & $3 \mathrm{~A} 4$ & None/(weak) 3A4, 3A5 & Like tipranavir & Like tipranavir \\
\hline Lopinavir & $3 \mathrm{~A} 4$ & None/3A4 & Like tipranavir & Like tipranavir \\
\hline Darunavir & $3 \mathrm{~A} 4(/ 5)$ & $?$ & Like pranavir (?) & Like tipranavir (?) \\
\hline \multicolumn{5}{|l|}{ FIs } \\
\hline Enfuvirtide & None & None/None & None (?) & All antiepileptic drugs \\
\hline
\end{tabular}

Abbreviations: NRTIs: nucleoside/tide reverse transcriptase inhibitors; NNRTIs: non-nucleoside reverse transcriptase inhibitors; PIs: protease inhibitors; FIs: fusion inhibitors.

Antiepileptic drugs: CBZ: carbamazepine; CLB: clobazam; CLN: clonazepam; DZP: diazepam; GBP: gabapentin; LEV: levetiracetam; LTG: lamotrigine; LZP: lorazepam; MDL: midazolam; OXC: oxcarbazepine; PB: phenobarbital; PHT: phenytoin; PGB: pregabalin; TGB: tiagabine; TPM: topiramate; VPA: valproic acid; ZOS: zonisamide.

\section{References}

Antinori A, Perno CF, Giancola ML, Forbici F, Ippolito G, Hoetelmans RM, Piscitelli SC (2005). Efficacy of cerebrospinal fluid (CSF)-penetrating antiretroviral drugs against HIV in the neurological compartment: different patterns of phenotypic resistance in CSF and plasma. Clin Infect Dis 41: 1787-1793.

Barton TL, Roush MK, Dever LL (1992). Seizures associated with ganciclovir therapy. Pharmacotherapy 12: 413-415.

Bates DE, Herman RJ (2006). Carbamazepine toxicity induced by lopinavir/ritonavir and nelfinavir. Ann Pharmacother 40: 1190-1195.

DiCenzo R, Peterson D, Cruttenden K, Morse G, Riggs G, Gelbard H, Schifitto G (2004). Effects of valproic acid coadministration on plasma efavirenz and lopinavir concentrations in human immunodeficiency virus-infected adults. Antimicrob Agents Chemother 48: 4328-4331.

Dore GJ, Correll PK, Li Y, Kaldor JM, Cooper DA, Brew BJ (1999). Changes to AIDS dementia complex in the era of highly active antiretroviral therapy. AIDS 13: 12491253.

Dou H, Birusingh K, Faraci J, Gorantla S, Poluektova LY, Maggirwar SB, Dewhurst S, Gelbard HA, Gendelman HE (2003). Neuroprotective activities of sodium valproate in a murine model of human immunodeficiency virus-1 encephalitis. J Neurosci 23: 9162-9170.

D’Silva M, Leibowitz D, Flaherty JP (1995). Seizure associated with zidovudine. Lancet 346: 452.

Kellinghaus C, Engbring C, Kovac S, Moddel G, Boesebeck F, Fischera M, Anneken K, Klonne K, Reichelt D, Evers S, Husstedt IW (2008). Frequency of seizures and epilepsy in neurological HIV-infected patients. Seizure 2007 Jul 5; 17: 27-33.

Langford TD, Letendre SL, Larrea GJ, Masliah E (2003). Changing patterns in the neuropathogenesis of HIV during the HAART era. Brain Pathol 13: 195210.

Lehrman G, Hogue IB, Palmer S, Jennings C, Spina CA, Wiegand A, Landay AL, Coombs RW, Richman DD, Mellors JW, Coffin JM, Bosch RJ, Margolis DM (2005). Depletion of latent HIV-1 infection in vivo: a proof-of-concept study. Lancet 366: 549-555.
Levy RM, Bredesen DE (1988). Central nervous system dysfunction in acquired immunodeficiency syndrome. $J A C$ quir Immune Defic Syndr 1: 41-64.

Liedtke MD, Lockhart SM, Rathbun RC (2004). Anticonvulsant and antiretroviral interactions. Ann Pharmacother 38: 482-489.

McArthur JC, Brew BJ, Nath A (2005). Neurological complications of HIV infection. Lancet Neurol 4: 543-555.

McGee B, Smith N, Aweeka F (2006). HIV pharmacology: barriers to the eradication of HIV from the CNS. HIV Clin Trials 7: 142-153.

Pascual-Sedano B, Iranzo A, Marti-Fabregas J, Domingo P, Escartin A, Fuster M, Barrio JL, Sambeat MA (1999). Prospective study of new-onset seizures in patients with human immunodeficiency virus infection: etiologic and clinical aspects. Arch Neurol 56: 609-612.

Price RW (1996). Neurological complications of HIV infection. Lancet 348: 445-452.

Schifitto G, Peterson DR, Zhong J, Ni H, Cruttenden K, Gaugh M, Gendelman HE, Boska M, Gelbard H (2006). Valproic acid adjunctive therapy for HIV-associated cognitive impairment: a first report. Neurology 66: 919921.

Siliciano JD, Lai J, Callender M, Pitt E, Zhang H, Margolick JB, Gallant JE, Cofrancesco J Jr, Moore RD, Gange SJ, Siliciano RF (2007). Stability of the latent reservoir for HIV-1 in patients receiving valproic acid. J Infect Dis 195(6): 833-6.

Temesgen Z, Cainelli F, Poeschla EM, Vlahakis SA, Vento $S$ (2006). Approach to salvage antiretroviral therapy in heavily antiretroviral-experienced HIV-positive adults. Lancet Infect Dis 6: 496-507.

Thomas SA (2004). Anti-HIV drug distribution to the central nervous system. Curr Pharm Des 10: 1313-1324.

Van den Brande G (2006). Kaletra independently reduces HIV replication in cerebrospinal fluid. Presented at the 13th Conference on Retroviruses and Opportunistic Infections 2006, Denver, CO, USA.

Venkataramana A, Pardo CA, McArthur JC, Kerr DA, Irani DN, Griffin JW, Burger P, Reich DS, Calabresi PA, Nath A (2006). Immune reconstitution inflammatory syndrome in the CNS of HIV-infected patients. Neurology 67: 383388. 